\title{
L'elève des bulletins scolaires : le spectre sémantique d’un modèle utopique
}

Original Study

Isabelle Monin

Université de Bourgogne Franche-Comté, Laboratoire CPTC et Université de Reims Champagne-Ardenne, laboratoire CIRLEP, France.

Received: August 2021; Accepted: November 2021

Résumé : La dichotomie entre le bon ou le mauvais élève semble réellement dépassée aujourd'hui. La manière de synthétiser les résultats scolaires et d'en rendre compte à l'écrit a positivement évolué malgré les routines restées ancrées dans les habitudes des enseignants. Dans cet article, nous proposons d'analyser l'orientation sémantique du mot élève lorsqu'il est employé dans les appréciations de livrets scolaires. Les concordances extraites d'un corpus attesté seront comparées à l'élève tel qu'il se trouve modélisé dans les textes officiels et par des chercheurs, ou tel qu'il se dessine dans les représentations des enseignants sur le terrain, débutants ou plus expérimentés. Nous montrerons que l'utilisation de ce mot et ses collocations quasi-systématiques révèlent des propriétés sémantiques qui trahissent la définition sous-jacente d'un élève idéal. S'agissant dans la réalité d'un individu en devenir, cet idéal est logiquement inaccessible, mais l'élève se trouve hissé suffisamment haut - du moins loué pour des traits de sa personnalité plus que pour son travail scolaire - et complimenté par le biais de sèmes afférents qui, paradoxalement, finissent par exclure l'enseignant de cette raison d'être pourtant nécessairement réciproque.

Mots-clés: analyse du discours, syntaxe, sémantique, lexicologie, communication Ecole-familles, bulletins scolaires, enseignement, évaluation

\section{THE STUDENT FROM SCHOOL REPORTS : THE SEMANTIC SCOPE OF AN UTOPIAN MODEL}

Abstract ${ }^{1}$ : The dichotomy between the good and the bad student seems really outdated today. The method used to summarize academic results and to report about them in writing has changed in a positive manner despite the routines still rooted in teachers' practices. In this paper, we will analyze the semantic orientation of the word "student" when it is used in school reports' assessments.

The concordances drawn from a certified body will be compared to the student as modelled in official guidelines and in educational sciences, or as sketched in the representations of the teachers in the field whether they are beginners or veterans. We will demonstrate that the use of this word and its almost systematic collocations in school reports show semantic qualities that betray an underlying definition of the perfect student, who is obviously unattainable since he or she is actually a future individual, the perfect student who is already highly praised. These praises are linked to his or her personality traits more than his or her school work strictly speaking. The perfect student is complimented through afferent semes which paradoxically end up excluding the teacher from this raison d'être although it is necessarily a two-way relationship

Keywords: Speech analysis, syntax, semantics, lexicology, communication between home and school, school reports, education, assessment

1 Thanks to Emmanuelle Bigerel and Heathcliff Sire for the help.

a Open Access. Open Access. @ 2021 Isabelle Monin, published by Sciendo.

(-.) This work is licensed under the Creative Commons BY 4.0 license 


\section{INTRODUCTION}

La désignation exclusive du bon vs mauvais élève semble pour le moins dépassée aujourd'hui. Le «bon petit élève » de 1930 retrouvé dans nos archives familiales, voire les laconiques bon ou excellent élève des bulletins des années 1940, lapidaires et apposant un mot à un rang, semblent tout à fait hors d'âge.

Même si l'usage de ce vocable n'est plus majoritaire à l'écrit, son utilisation demeure porteuse d'un champ sémantique récurrent et singulier vis-à-vis de la place centrale qu'il est censé occuper en contexte. Expliquons-nous : ce terme, en apparence trivial, fait partie du lexique ordinaire du monde de l'Ecole, où l'enfant des familles devient élève par le biais d'une translation à la fois physique et intellectuelle, plus ou moins étanche, signant tacitement, par son inscription, un engagement de conformité avec des valeurs socioculturelles implicitement partagées. Sa définition, sa caractérisation et sa référence, en contexte scolaire, demeurent pourtant complexes à circonscrire, car cela dépend des points de vue qui l'emploient. En effet, son usage vise-t-il une fonction, la dénomination localisante d'un individu au sein d'un établissement scolaire et d'un processus tacite d'apprentissage à l'intérieur de ce dernier, ou un concept générique et idéal, porté plus ou moins sciemment par I'Institution, auquel tout individu scolarisé doit à tout prix finir par correspondre?

Cette question est loin d'être anecdotique, car c'est ce qu'implique le terme de Socle des programmes sur lequel l'élève peut - métaphoriquement certes - s'appuyer pour s'élever... avec ses enseignants, mais jusqu'où ? Est-il possible d'atteindre le haut de l'échelle? Selon la formule de Benveniste, «le langage re-produit la réalité », c'est-à-dire que « celui qui parle fait renaître par son discours l'événement et son expérience de l'événement. » Nous observerons donc quelle « réalité » se trouve « re-produite » par les enseignants lorsqu'ils rédigent, de manière nécessairement synthétique, un bilan périodique des élèves qu'ils ont en responsabilité, et quel miroir - formant ou déformant - ils tendent à leurs parents.

\section{PRÉSENTATION DU CORPUS}

Guiraud (1960) nous indique qu' « un mot se définit littéralement par l'addition de ses emplois ». C'est la raison pour laquelle nous avons choisi d'observer l'utilisation du mot élève dans les livrets scolaires ${ }^{2}$, en parallèle avec, d'une part, sa définition lexicographique dans plusieurs dictionnaires, celle proposée en formation par un échantillon d'enseignants, son utilisation dans le Socle Commun de Compétences, Connaissances et de Culture ${ }^{3}$, et un article de recherche connu des étudiants de Master MEEF. Nous observerons les convergences et divergences sémantiques à l'intérieur de ces différents discours réalisés dans le même domaine d'activité.

\subsection{MÉTHODOLOGIE PRAGMATIQUE}

Le corpus d'étude est une compilation de 2724 livrets provenant d'une cinquantaine d'écoles, de collèges et de lycées différents en France métropolitaine, sur une dizaine d'années. Partitionné par niveau, année et sexe de l'élève, afin de pouvoir observer, le cas échéant, une différence de traitement entre les filles et les garçons ${ }^{4}$.

Nous nous sommes appuyée sur l'ouvrage récapitulatif des méthodes d'Analyse de données textuelles de Lebart, Pincemin ${ }^{5}$ et Poudat (2019), et avons utilisé le logiciel TXM pour identifier les cooccurrents les plus fréquents du pivot élève.

\subsection{UN VOCABLE SOUS LES LOUPES THÉORIQUES}

Ce lexème a la caractéristique pratique d'être épicène et utilisé avec une différence de nuances référentielles dans des documents divers issus de la classe sémantique //école//. Cette lecture nous a amenée à observer l'ensemble des items qui composent sa valeur sémantique en fonction du contexte et du point de vue, valeur sémantique renforcée par sa représentation syntaxique.

Nous nous adosserons à la méthode d'analyse décrite par Mortureux (1997, 64-65) en analysant la structure syntagmatique de ce nom, et les rôles sémantiques qui lui sont liés, afin d'en dégager le « nœud de relations sémantiques [qu'il] instaure », car comme le souligne Krazem dans une étude portant sur un autre vocable (1996), « certains sèmes sont directement issus des environnements linguistiques (déterminations, épithètes...). D’autres sont saisis moins directement ».

Si les traits sémantiques du devenir élève des discours institutionnels semblent à la fois antinomiques et pléonastiques, l'individu enfant en phase de développement physique et intellectuel est inscrit dans un établissement pour que ce dernier complète l'éducation reçue à la maison, et son accomplissement en tant que tel se place sous la responsabilité du corps enseignant. Ce devenir élève est donc, non pas de la responsabilité des familles, mais bien une compétence à acquérir à l'école, mais là

2 Le Livret Scolaire Unique (désormais LSU) comporte maintenant les bilans périodiques, anciennement nommés bulletins scolaires dont nous gardons ici l'usage par commodité terminologique culturelle.

3 Désormais S4C ou Socle.

4 Par exemple, la reproduction éventuelle de stéréotypes de genre intégrés dans les attentes des enseignants et observables dans les formules utilisées.

5 Nous remercions à cette occasion Bénédicte Pincemin et Serge Heiden pour leur aide précieuse en juillet 2020 concernant le choix crucial de la méthodologie de préparation du corpus pour son import dans TXM 
où son emploi en français est fascinant, c'est qu'un élève dont on dit qu'il est mal élevé n'est jamais désigné ainsi à cause d'une carence pédagogique d'un enseignant ${ }^{6}$.

\section{LE GENRE DU BULLETIN SCOLAIRE : L'ÉLOGE OU LE BLÂME DES FAMILLES}

Dans son ouvrage consacré à l'évaluation des élèves, Roegiers (2004) indique que "Le bulletin scolaire constitu[e] le trait d'union principal entre l'école et la société », une sorte de verdict régulier qui sert, non seulement de bilan d'une période achevée, mais idéalement de marchepied en vue d'une progression à venir, tout en prenant soin d'asseoir une autorité institutionnelle nécessaire à sa légitimité. Ce document n'est donc pas seulement une trace écrite officielle du parcours de l'élève, mais il prend également la forme d'une lucarne qui permet aux familles d'avoir un regard sur le positionnement de leur enfant vis-à-vis des savoirs enseignés sur une période donnée, ainsi que sur son comportement à l'école.

\subsection{UN RÔLE INVOLONTAIRE DE COUPERET RÉGULIER}

Selon le Dictionnaire historique de la langue française d'A. Rey ainsi que dans le Larousse de l'ancien français, derrière le lexème bulletin apparaissent les traits sémantiques communs de /synthèse/, /évaluative/, /qui vient d'une autorité institutionnelle/. L'appellation actuelle livret conserve ces traits sémantiques, mais tend à considérer plus explicitement le parcours scolaire de l'élève comme un tout composé de plusieurs pages correspondant à des étapes, et mettant en valeur une progression.

Maingueneau (2000 [1998]) en parle comme d'un « discours contraint inséré dans une situation sociale de communication assez bien définie », et le caractérise comme une « routine que suffit d'ailleurs à évoquer cette phrase particulièrement emblématique du bulletin qu'est le fameux Peut mieux faire », formule-titre d'un article de S. El Hadi (2011).

Pour cette dernière, qui a étudié les routines liées à ce support avant la dernière réforme mais dont les résultats sont toujours d'actualité, il est « un commentaire évaluatif ou appréciatif censé rendre compte de la valeur scolaire du travail fourni par un individu clairement désigné (...) posé en vis-à-vis d'un ensemble de données chiffrées, les notes, qu'il doit éclairer en en fournissant une interprétation. » Par conséquent, il doit être personnalisé, au regard des exigences institutionnelles de différenciation, et bienveillant, au même titre que l'évaluation dans le sens de /donner une juste valeur à/. Ainsi, cette formulation synthétique se doit d'être conforme aux exigences d'enseignement : se situer par le discours dans la Zone Proximale de Développement ${ }^{7}$ de l'élève, théorisée par Vygotski (1985). II apparaitrait ainsi presque évident que ce bulletin scolaire s'adresse à l'élève, ce qui n'est pas toujours le cas.

\subsection{UNE POLYPHONIE UNILATÉRALE... EN APPARENCE}

Une caractéristique peut-être particulière à la communication entre l'Ecole et les familles, c'est que la dichotomie entre énoncés constatifs et énoncés performatifs au sens d'Austin, n'est pas si nette, et pragmatiquement, le constatif de l'appréciation générale rédigée collectivement peut avoir une valeur performative, ce qui nous renvoie au discours épidictique d'Aristote, par le biais de trois actions qui correspondent aux accessits : féliciter et encourager, ou blâmer, sur une échelle allant de non-accessit à la mise en garde, puis à l'avertissement, une sanction inscrite au dossier scolaire de l'élève.

\subsubsection{UNE PLURALITÉ D'ÉNONCIATEURS AUX FORMULES STANDARDISÉES}

La relation énonciative du bulletin scolaire est particulièrement complexe. En effet, si le bilan périodique se veut simple et synthétique, cela ne peut se faire qu'au prix de la pluralité énonciative, chaque enseignant et personnel de direction ayant une possibilité d'expression. En revanche, aucune réponse n'est attendue de la part des familles ${ }^{8}$, à moins que l'on en attende une réponse non-verbale. En réalité, ce bilan ne s'adresse qu'indirectement à l'élève ce qui est finalement logique puisqu'ils vivent ensemble ces moments scolaires qui se régulent en direct, et que les personnes qui les découvrent a posteriori sont les parents. Ce qui signifie que le bilan périodique attend tout de même une réponse, sans l'exprimer explicitement sauf en cas d'avertissement, sanction disciplinaire - mais qui prendra la forme d'une constance ou d'une modification de l'attitude comportementale et/ou face au travail, grâce aux parents redevenus responsables de l'autorité sur l'enfant, puisque ce discours a vocation à dépasser le cadre spatiotemporel de l'école.

$6 \mathrm{Si}$ «mal éduqué » existe en français, mais plutôt pour parler d'animaux domestiques, la formulation «mal enseigné » est utilisée à la forme passive, passant sous silence le sujet actif, et jamais pour qualifier un nom humain.

7 Pour approfondir à propos de la ZPD, voir Vygotski dans Pensée et langage (1985), où ce concept incite notamment les pédagogues à situer - et adapter - leur enseignement en fonction de la distance entre ce que l'élève sait déjà faire seul et ce qu'il ne saura pas réaliser sans aide, de l'enseignant et/ou de ses pairs : cette notion ne se concentre pas seulement sur les acquis passés mais est tournée vers l'avenir, en identifiant une zone abstraite de potentialité de progression spécifique à chaque élève qui encourage la collaboration entre eux pour une réelle élévation sociocognitive.

8 Excepté lors de brefs RDV individuels, de type réunions parents-professeurs. 


\subsubsection{L'ÉLÈVE DÉSIGNÉ EXCLUSIVEMENT À LA 3e PERSONNE}

Dans l'ensemble des phrases comportant le nom élève ${ }^{9}$, nous pouvons constater que l'enseignant désigne l'enfant à la 3e personne, montrant ainsi que ces phrases s'adressent en réalité à ses parents. Ainsi, les emblématiques suivants en (1), (2) et (3), que l'on ne lira pas sous la forme (4) alors que la formulation est grammaticalement correcte ${ }^{10}$ :

(1) Bon travail et bonne participation, élève sérieuse et agréable.

(2) Un premier trimestre parfaitement réussi. Raphaël est un élève sérieux et travailleur. Je le félicite et l'invite à continuer ainsi par la suite.

(3) Adrien est un élève sérieux et motivé avec de bonnes capacités.

(4) \# (Morgane,) tu es/vous êtes une élève très sérieuse qui fait beaucoup d'efforts.

Lorsque le propos n'est pas complètement positif et que l'enseignant attend une modification du comportement, il reste à la 3e personne. Par conséquent, l'énoncé ne peut être simplement constatif et nous pouvons les interpréter comme des actes de langage ${ }^{11}$ indirects appelant implicitement une intervention de l'autorité parentale :

(5) Bon trimestre. Jehan est un élève qui a de bonnes connaissances et qui travaille sérieusement. Cependant, il a parfois du mal à canaliser son énergie en classe.

(6) Pierre est un élève agréable. II s'applique dans son travail. C'est bien! Mais il n'est pas toujours attentif lors des activités collectives.

(7) Aymeric est un élève investi qui a de très bonnes capacités. Cependant le manque de soin, la précipitation et les bavardages nuisent parfois à son travail.

En revanche, à l'intérieur de l'appréciation, l'enseignant ajoute parfois une phrase à l'impératif ou un tutoiement, que l'on interprète nécessairement comme s'adressant à l'enfant, cette familiarité n'étant pas concevable dans la relation enseignant/parents, quand bien même ils se connaitraient personnellement. Les deux destinataires directs sont donc pris en considération dans une même appréciation, même si les attentes sont différentes :

(8a) Camille est une élève sérieuse, volontaire, autonome et investie dans la vie et les projets de la classe. Elle a obtenu de bons résultats durant ce premier trimestre ! C'est bien, continue ainsi !

(9) Bon travail. Elève très agréable. Continue comme ça.
(10) C'est un excellent semestre. Emelle est une élève mature, sérieuse et appliquée. Pas une seule ombre au tableau ! Je t'adresse toutes mes félicitations !

(11) Bon semestre. Marie a quelquefois peur de se tromper. II n'y a pas de problème d'apprentissage. Elève souriante et discrète. Bravo. Continue.

(12) Élève dynamique, un peu trop parfois ;)

Ces occurrences montrent que l'on félicite l'enfant pour son respect à la conformité attendue par la fonction élève, mais sans l'inscrire lui-même dans cette énonciation. Pour cela, on a besoin d'une phrase supplémentaire, ce qui peut être perçu, si l'on extrapole à partir des $\mathrm{AL}$, comme une manière indirecte de féliciter les parents d'avoir su installer les fondements de cette appartenance à la norme ${ }^{12}$.

L'exemple (12) est cependant quelque peu différent et observé pour l'instant uniquement à l'école primaire : il est déjà assez rare de voir se réaliser des émoticônes dans les écrits des enseignants, surtout dans des documents officiels, alors malgré le déterminant zéro ${ }^{13}$ qui implique nécessairement une 3e personne, le smiley ne peut s'adresser qu'à l'enfant. L'interprétation de connivence qui découle du clin d'œil montre qu'ici, l'enseignante met en valeur une qualité, et malgré le constat des débordements potentiels qui lui sont liés, n'attend aucune intervention de la part des parents, mais prend en charge l'entière responsabilité de sa gestion de classe.

\subsection{SYNTAXE DE L'ÉLÈVE}

La construction syntaxique des bilans scolaires, particulièrement ceux qui font apparaître le $N_{e^{\prime}}$ est très routinisée. Seul change le nombre de phrases, ou la longueur de l'appréciation, qui consiste en un développement seIon que l'élève, nommé ou non, possède des qualités qui correspondent au comportement normé attendu, ou est invité à travailler certains points pour s'en rapprocher : autrement dit, une formulation positive mais..

\subsubsection{UN GN ÉTENDU AVEC UNE DÉTERMINATION INDÉFINIE}

Comme nous le constatons dans l'ensemble des occurrences sélectionnées pour leur fréquence représentative, la routine liée à l'utilisation du $N_{\mathrm{e}}$ veut que le nom soit toujours introduit, soit par le det $\varnothing$, soit par l'article indéfini un, réciproquement permutables selon que l'enseignant

\footnotetext{
9 Désormais $N_{\mathrm{e}}$.

10 Nous empruntons à la sémantique formelle (Roussarie, 2017) le signe dièse, et non l'astérisque qui indiquerait une agrammaticalité, pour indiquer un énoncé issu de nos manipulations de phrases du corpus, et qui serait inadéquat contextuellement ou en tous les cas non attesté.

11 Désormais AL.

12 Conformité à la norme attendue voire conditionnement social dans « Elève souriante et discrète. Bravo. Continue ». A noter que sur l'ensemble du corpus élargi au-delà du nom élève, les remarques - en termes de renforcement positif ou négatif - liées au sourire, la discrétion et la bonne humeur, non qu'il s'agisse à proprement parler d'une injonction, étant souligné majoritairement chez les filles, à hauteur de $80 \%$.
}

13 Désormais det $\varnothing$. 
aura fait le choix d'un énoncé verbal ou non. Soit en (3)

(3) Adrien est un élève sérieux et motivé avec de bonnes capacités.

=> Elève sérieux et motivé avec de bonnes capacités.

Beyssade (2020) nous rappelle au sujet des implicatures que pour Heim (1982), «les indéfinis introduisent une variable X parmi d'autres » et que pour Hawkins (1991), l'indéfini un $N$ implique « qu'il existe plus d'une entité dans l'univers du discours ayant la propriété $N$ », soit, en (8a) notamment, Camille est une élève, mais pas n'importe laquelle, une élève sérieuse, parmi d'autres élèves (sérieux ?), volontaire, parmi d'autres élèves (volontaires ? sérieux et volontaires ?), autonome (parmi d'autres élèves autonomes? sérieux, volontaires et autonomes?) etc.

Ainsi, cette utilisation du $N_{\mathrm{e}}$ vise à conférer au référent du nom propre ${ }^{14}$ son statut d'élève en ce qu'il est représentatif d'un type d'élève ou doit s'en rapprocher en correspondant à tels ou tels critères. En effet, ce GN est toujours étendu en contexte, ce qui tend à comparer l'élève dont on fait le bilan aux autres membres de son groupe-classe, mais pas seulement, par extension par rapport aux types d'élèves existant dans notre culture collective voire notre imaginaire collectif. En effet, on n'observera pas :

(8b) \# Camille est une élève.

(8c) \# Camille est L'Elève.

(8d) \# Camille est l'élève sérieuse, volontaire, autonome et investie dans la vie et les projets de la classe.

(8e) ? \# Camille est l'élève sérieuse, volontaire, autonome et investie dans la vie et les projets de la classe dont chaque enseignant peut rêver.

(8f) \# Camille n'est pas l'élève sérieuse, volontaire, autonome et investie dans la vie et les projets de la classe dont chaque enseignant peut rêver.

(8g) \# Camille n'est pas une élève sérieuse, volontaire, autonome et investie dans la vie et les projets de la classe.

Et plus rarement ce type de formulation :

(13) Kevin est le seul élève à ne pas mavoir rendu sa rédaction.

(14) Insuffisant. Élève bavard, peu attentif et qui n'a pas tenu compte des conseils.

En (8b), l'indéfini renverrait à l'ensemble de la classe élève : en effet, puisque le genre du bulletin scolaire fait préexister l'individu, le rôle de construction d'existence de l'indéfini n'est pas convoqué. Si Camille a un bulletin, elle est élève en tant qu'inscrite dans l'établissement, il ne s'agit pas d'une information mais d'une présupposition liée à ce sème inhérent ${ }^{15}$. De même qu'en (8c), on attendrait une complémentation en $\left[\mathrm{le}+N_{\mathrm{e}}+\right.$ de $\left.\mathrm{X}\right]$ qui expliciterait le trait sémantique de la relation à un ascendant humain, mais l'article défini implique davantage le complément du nom en tant que trait distinctif entre plusieurs $N_{\mathrm{e}^{\prime}}$ soit parmi plusieurs $N_{\mathrm{e}^{\prime}}\left[\mathrm{A}=N_{\mathrm{e}}+\right.$ de $\left.\mathrm{X}\right]$, $\left[\mathrm{B}=N_{\mathrm{e}}+\right.$ de $\left.\mathrm{Y}\right],\left[\mathrm{C}=N_{\mathrm{e}}+\right.$ de $\left.\mathrm{Z}\right]$, donc n'est jamais lisible dans notre corpus, excepté en (13), qui possède une implicature exclusive des autres du point de vue de l'enseignant avec sa structure en $\left[K=l e+\right.$ seul $+N_{e}+$ Pred $\left(\neq\right.$ tout autre $\left.N_{\mathrm{e}}\right)$ ]. Une seule possibilité d'apparition (non attestée) : si l'article défini était en majuscule d'insistance du type (8c), qui impliquerait par inférence l'élève idéale unique qu'il n'est pas besoin de décrire car elle contiendrait en [ $\left.L ' N_{\mathrm{e}}\right]$ tous les sèmes afférents sous-entendus, et donc partagés par tous, et incarnerait en quelque sorte «L'Emile » des enseignants, excluant tous les autres.

Dans l'exemple (8d), l'article défini, en tant qu'exclusif des autres s'avèrerait improbable dans ce contexte, à moins d'une prédication verbale du type (8e), mais l'exemple n'est pas attesté et, en raison de la comparaison sous-jacente, serait plutôt jugé trop élogieux pour l'élève et donc trop méprisant vis-à-vis des autres ${ }^{16}$. La forme négative des exemples (8f) et (8g), en ce qu'elle nie toute qualité à l'élève, ou en tous les cas souligne cette absence de qualités, s'avèrerait particulièrement violente et n'est pas attestée.

D'autre part, il arrive aussi souvent que l'appréciation soit plus concise, et dans ce cas, le $N_{\mathrm{e}}$ apparaît dans une phrase averbale avec omission du référent, jugé suffisamment saillant pour être compris par l'ensemble des destinataires ${ }^{17}$, le sujet est implicite et identifié par la situation d'énonciation, voir ici en (1) (9), (12) et de toute manière convoqué anaphoriquement en (11), exemples que nous répétons ici :

(1)Bon travail et bonne participation, élève sérieuse et agréable.

(9) Bon travail. Elève très agréable. Continue comme ça.

(11) Bon semestre. Marie a quelquefois peur de se tromper. II n'y a pas de problème d'apprentissage. Elève souriante et discrète. Bravo. Continue.

(12) Élève dynamique, un peu trop parfois ;)

(14) Insuffisant. Élève bavard, peu attentif et qui n'a pas tenu compte des conseils.

En cas de phrase averbale, l'absence de détermination est nécessitée pour des raisons syntaxiques ${ }^{18}$ parce qu'il n'est pas besoin d'actualiser : il y a une relation directe entre l'énonciateur et le prédicat, et cette déicticité est favorable aux énoncés subjectifs. Majoritairement, dans ces occurrences, l'expansion met en évidence des qualités ${ }^{19}$, sauf dans un seul cas en (14),

\footnotetext{
14 Désormais NP.

15 L'ensemble des traits sémantique relevés sont synthétisés en Fig.1, § 3.2.

16 Cette interprétation serait comparable avec les déterminants possessif \#mon élève et démonstratif \#cet élève que nous ne pouvons développer ici.

17 Nous rappelons que le bilan périodique est nominatif et individuel. Même en cas d'erreur de prénom de la part d'un enseignant sur ce document, l'élève et ses parents savent sans ambiguïté qu'il est désigné.

18 Nous ne les développerons pas ici.

19 II subsiste tout de même des cas limites à explorer avec une vision de l'ensemble du corpus non focalisée
} 


\section{L'elève des bulletins scolaires : le spectre sémantique d'un modèle utopique}

exclusivement négatif.

Pour ce qui est du det $\varnothing$, constitutif de l'énoncé averbal, il est restituable par l'article indéfini précédemment décrit suivi du verbe être et du sujet identifiable dans la situation d'énonciation : Elève agréable -> NP est un ou une élève agréable.

Buvet (2009) nous indique que les noms qui reçoivent une détermination $\varnothing$ sont « des substantifs dénotant des propriétés suffisamment permanentes pour identifier des humains par rapport à d'autres », notamment des noms liés à des «fonctions », ce qui confirme notre interprétation, à savoir que le $N_{\mathrm{e}}$ revêt le même emploi et que l'élève réel y est assigné en tant qu'appartenant à un type d'élève, tout en accentuant la subjectivité par l'omission d'un sujet nommé.

\subsubsection{DES EXPANSIONS DU NOM PRÉDICATIVES}

Dans nos occurrences, le GN étendu contient l'information essentielle de la phrase, mise en relation avec le sujet implicite ou explicite par le biais de la copule, exprimée ou sous-entendue, être (Desclès 2008) : "Pour remplir cette fonction de mise en relation, la copule est devient un opérateur de mise en relation (appelé alors relateur) qui construit un Prédicat (logique) appliqué ensuite au Sujet ». L'enseignant indique une caractéristique du sujet, permanente ou du moins durative. En effet, ce prédicat indique une propriété vraie pour l'énonciateur au moment où elle est écrite, une propriété attribuée à un individu, et l'assignation à cette propriété se fait au présent ${ }^{20}$.

Nous l'avons vu avec les tests en (8b), comme indiqué dans Riegel et al., « la suppression d'un modifieur déterminatif modifie la valeur référentielle du GN » ( $p$. 343). Ainsi, nous sommes bien confrontés à un rapport déterminatif qui restreint l'extension du nom, ici, par ajout de modifieurs, l'extension de la «classe-type ${ }^{21}$ d'élève » employée relativement à une classe générique contextualisée, comme le prouve la possibilité pour chaque occurrence de se pronominaliser en en partitif [NP en est un ou une] (p. 426) : l'élève désigné appartient à cette classification qualitative virtuelle.

En effet, si l'on observe à présent la construction de tout ce qui modifie le $N_{\mathrm{e}}$, la constellation de ses expansions, on remarque que cette addition d'informations qualitatives tendra à réduire les possibilités de correspondance des autres élèves à l'élève modèle qui se dessine sémantiquement. Nous n'ignorons pas l'isotopie positive, ou les indications pour l'atteindre, lisibles dans l'ensemble de l'appréciation, mais la structure syntaxique du GN au $N_{\mathrm{e}}$ des énoncés précédents nous indiquent quelques routines en (15):

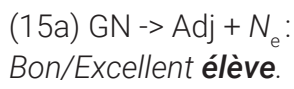

Nous remarquons tout d'abord que les seuls adjectifs antéposés sont les traditionnels bon et excellent $N_{\mathrm{e}^{\prime}}$ le premier potentiellement modifié par les adverbes très ou assez, que nous trouvons encore, quoique plus rarement. Ce sont les résultats, le trimestre, le bilan qui sont majoritairement qualifiés de bon ou excellent, dans la majorité des bulletins, et non l'élève lui-même, la focale visée par ces qualificatifs s'étant visiblement déplacée du jugement sur l'individu à son rapport aux savoirs, ou celui-là a revêtu une autre forme.

Quoi qu'il en soit, cette antéposition systématique de l'adjectif excellent, certes routinière, et qu'il s'agisse du bilan ou du $N_{e}$ a la particularité d'ajouter au nom désigné une valeur affective, malgré l'apparente neutralité du laconique excellent élève.

Nous observons d'autre part que les adjectifs qualificatifs postposés au $N_{\mathrm{e}}$ sont souvent les mêmes, parfois modifiés par un adverbe ou un groupe nominal prépositionnel ${ }^{22}$ qui apporte une précision localisante pas forcément utilitaire car aisément inférable, mais qui semble appelée parfois par certains adjectifs du type investi ou investie dans la vie de la classe attentif ou attentive en classe, dynamique à l'oral, qui généralement ne restent pas en emploi absolu mais allongent crescendo la quantité syllabique de la fin de la phrase.

Ces épithètes adjectivales, tout comme les

sur le $N_{\mathrm{e}}$ : si le qualificatif dynamique est connoté positivement, calme également, l'énergie est à canaliser. De même qu'en fonction du contexte, discret ou discrète est positif mais peut générer une injonction, à inférer de la présence du mot : une requête de participation orale. Cette observation fera l'objet d'une analyse de scalarité précise et peutêtre spécifique à l'épistolaire éducatif.

20 L'analyse du verbe être et de l'utilisation du présent de l'indicatif sera réalisée indépendamment du $N_{\mathrm{e}}$ sur l'ensemble du corpus et élargie aux autres supports de l'épistolaire éducatif.

21 Nous préférons utiliser classe-type plutôt que classe, pour éviter l'ambiguïté sémantique (malgré le trait commun tout à fait à propos).

22 Désormais GP. 
propositions subordonnées relatives ${ }^{23}$, ont une fonction équivalente, mais on remarque certaines habitudes de formulation : les modifieurs sont juxtaposés, cumulables jusqu'à une certaine limite ${ }^{24}$, la dernière expansion étant coordonnées par et/mais, la PSR étant toujours en fin de phrase, pour les mêmes raisons prosodiques qu'indiquées précédemment.

Nous avons identifié une dernière catégorie de modifieurs, les noms épithètes en (16a) et (17), qui, comme indiqué dans Riegel et al., assignent au $N_{\mathrm{e}}$ une qualité saillante, ici « envisagée métaphoriquement » (p. 345) :

(16a) Un bon niveau général et surtout une excellente participation en cours. Mélissa est une élève moteur de la classe.

(17) Mathis est un élève intéressant, intéressé, inteIligent et cultivé. Il est pour les autres un élève ressource qui permet à tous de progresser. II faut continuer ainsi.

$(16 b)$ * (...) Mélissa est une élève motrice/turbine/ réacteur/ ? locomotive de la classe.

(16c) * (...) Mélissa est une/l'élève pot d'échappement de la classe.

L'utilisation du nom épithète moteur est relativement figée et mixte ${ }^{25}$ : on envisagerait difficilement, en tous les cas à l'écrit ${ }^{26}$, un paradigme synonymique de type (16b) élève turbine ou élève réacteur, éventuellement élève locomotive, mais sa connotation vieillie et ses 4 syllabes le rendent difficilement attestable. Comme vu précédemment, il s'agirait d'un exemplaire unique donc on utiliserait de préférence l'article défini, ce qui n'est pas le cas du nom épithète moteur, qui implique, grâce à l'indéfini, qu'il peut y avoir plusieurs élèves correspondant à ce prototype.

D'autre part, autant cette comparaison est positive, en ce qu'elle met en valeur des qualités de dynamisme et de d'influence positive sur le groupe classe, autant l'utilisation d'une métaphore de même champ lexical mais de connotation inversée du type (16c) ${ }^{27}$ serait bien entendu du plus mauvais goût en termes d'humour sur un bulletin scolaire.

\section{DU RÉEL AU VIRTUEL, UN STATUT QUI SE MÉRITE}

Selon Mortureux (1997, 72), «la dissociation observée entre les noms et les choses permet d'exprimer économiquement les points de vue variés sur la réalité », c'est pour cela que nous avons choisi d'observer le $N_{\mathrm{e}}$ par le prisme de ses manifestations diverses, synthétisées dans la Fig. 1 infra.

Nous constatons que dans les discours propres à l'éducation, la notion même d'élève est différente : il ne s'agit pas toujours du même élève, qu'il s'agisse d'une représentation virtuelle, une modélisation théorique, ou la manière dont les enseignants réalisent où construisent une figure d'élève dans les bulletins scolaires.

\subsection{L'ÉLĖVE DES DICTIONNAIRES}

Dans ce paragraphe, nous nous sommes penchée sur les définitions virtuelles du lexème élève tel qu'il est visible dans plusieurs dictionnaires ${ }^{28}$, dans le but de faire apparaître ses traits sémantiques inhérents, qui seront de fait présupposés dans le contexte socioprofessionnel qui nous intéresse. Le Larousse en ligne et le TLFi, notamment, proposent plusieurs acceptions, que nous pouvons résumer en sèmes micro-génériques, à savoir /jeune être humain/, /qui reçoit un enseignement d'un ascendant/, /inscrit dans un établissement spécialisé/, /pour progresser/. Le sème /appartenant à un groupe/ n'est cependant pas systématique.

\subsection{L'ÉLĖVE DES BULLETINS SCOLAIRES}

Lorsque nous nous penchons sur le sens lexical d'un mot, Mortureux (1997, 70-71) nous invite à retenir son « caractère décomposable et son organisation différentielle » : «le sémème est le signifié d'un lexème, dont l'organisation interne revêt la forme d'une collection ». Dans le corpus retenu pour cette étude, où nous utilisons la terminologie et la signalétique de Rastier (2009 [1987]), nous avons pu identifier un certain nombre de traits sémantiques récurrents pour le $N_{\mathrm{e}}$ qui, au-delà du classème, i.e. les traits constitutifs présents dans chaque support - soit explicitement, soit présupposés, soit par implicature - sont représentatifs de ce qui est attendu par le corps enseignant vis-à-vis de l'enfant réel.

Si nous avons élargi la focale, c'est surtout parce que l'environnement syntaxique à l'œuvre autour du $N_{\mathrm{e}}$ dans les appréciations scolaires est, nous l'avons vu, très routinisé, et que le champ sémantique qui s'en dessine tend à préciser les critères à remplir pour obtenir le sacré graal de la dénomination élève. Nous avons synthétisé l'ensemble des traits récurrents en sèmes micro-génériques en Fig. 1. Si les traits paraphrasés dans les dictionnaires sont obligatoirement inhérents ou présents par

23 Désormais PSR.

24 Limite de quatre expansions.

25 L'absence d'accord au féminin en *élève motrice (16b) prouve qu'il s'agit du nom et non de l'adjectif.

26 La métaphore avions de chasse pour désigner des élèves particulièrement performants et rapides est attestée à l'oral, mais dans des conditions de connivence dans la relation élève/enseignant ou enseignant/enseignant que ne supporte pas l'écrit, surtout en direction des parents, qui pourraient juger cette déshumanisation maladroite, quoique positive.

27 Occurrence inspirée d'un site humoristique d'enseignants qui, comme beaucoup d'autres sur la même thématique, tourne en dérision les routines et le vocabulaire spécifique du monde éducatif.

28 Le panorama lexicographique méritera d'être développé ultérieurement. 


\section{L'elève des bulletins scolaires : le spectre sémantique d'un modèle utopique}

présuppositions dans les autres perceptions, même modélisantes, reproduits dans la partie gauche du tableau, nous avons relevé tous les co-occurrents « nécessaires à l'élaboration du sens et condition de l'interprétation » (Mayaffre 2008), c'est-à-dire toutes les expansions et les injonctions indirectes contextuelles et systématiques au sein de notre corpus. Nous avons reproduit dans la partie droite du tableau les traits afférents observables dans les appréciations scolaires comportant le $N_{\mathrm{e}^{\prime}}$ en comparaison avec leur présence éventuelle au sein des autres discours, par présupposition ou par implicature en cas de présence non-explicite du sème.

Ainsi, que ce soit dans nos occurrences choisies supra et ce que nous avons observé dans la globalité du corpus, nous pouvons constater que l'enfant ou adoles- à bon escient. II est, de plus, motivé, volontaire, attentif et concentré en permanence, manifeste son intérêt pour le cours, et se montre de préférence agréable, humble, souriant ${ }^{29}$, et ne s'oppose pas à l'enseignant $\left(N_{\mathrm{e}}\right.$ positif) positive, sympathique, attachant/attachante, serviable voire dévoué/dévouée).

Ce qui est également apprécié, ce sont les traits sémantiques soulignant, d'une part, la mise en valeur de ses habiletés cognitives et le milieu enrichi de son cadre socioculturel (intelligence, culture générale, politesse, compréhension du rôle de l'Ecole, bonnes capacités ou bon/ excellent potentiel) - autrement dit, tout ce qui n'a pas été enseigné par le professeur qui rédige ces phrases et d'autre part, le fait d'être autonome voire remplissant un rôle d'assistant de l'enseignant qui bénéficie de ces

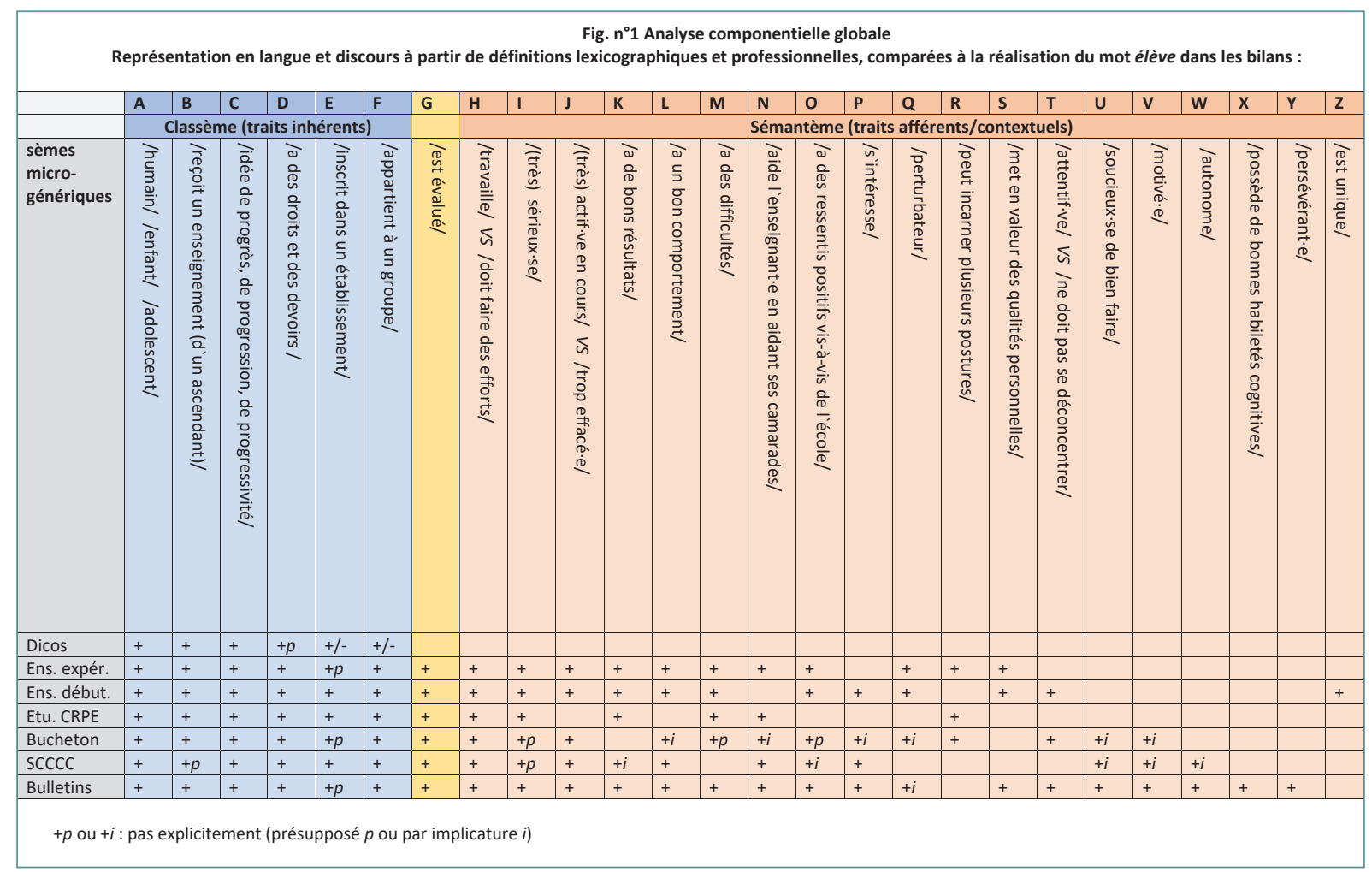

cent /inscrit dans un établissement scolaire/ qui mérite le statut d'élève dans ses bilans périodiques, est entouré syntaxiquement d'expansions du nom qui portent des traits sémantiques vériconditionnels et constatifs de l'attitude en classe sur la durée, mais particulièrement subjectifs, malgré l'objectivité apparente de l'absence de marqueurs énonciatifs. En effet, l'élève idéal, tel qu'il est décrit ou ambition à atteindre au vu des conseils ou plutôt des directions d'attitudes à ajuster, se doit d'être irréprochable sur tous les plans et rester d'humeur égale. Il travaille régulièrement, soigneusement et qualitativement, est sérieux en toutes circonstances, fournit des efforts si le travail est plus difficile ou s'il rencontre des difficultés, obtient de bons résultats, reste calme sans être effacé, tout en étant dynamique et participe[r] à l'oral avantages in situ, qu'il soit moteur ou ressource pour les autres. En réalité, au milieu de tous ces critères positifs, l'enfant réel a l'air d'être pleinement responsable de sa réussite... ou de ses insuffisances (Fig.2). Mais cette responsabilité se trouve tout de même diluée car, nous l'avons vu, la troisième personne implique que les parents sont les principaux destinataires de ces phrases spécifiques, porteuses de la valeur performative adressée à leur enfant.

\subsection{L'ÉLÈVE DES MODÈLES THÉORIQUES}

29 Rappel : majoritairement souligné chez les filles à hauteur de 80\%. 


\subsubsection{L'ÉLĖVE DU SOCLE COMMUN DE COMPÉTENCES, DE CONNAISSANCES ET DE CULTURE}

Si le référentiel des compétences professionnelles de 2013 enjoint les enseignants à prendre en compte la diversité des élèves, et utilise le vocable exclusivement au pluriel avec une détermination définie, l'élève du S4C n'est pas réalisé de la même manière.

En effet, alors que ce n'est pas systématique dans les programmes, le B.O de 2015 utilise exclusivement ${ }^{30}$, sur les 8 p. qui le composent, 57 fois l'élève au singulier avec une détermination définie, et développe les compétences à acquérir sous forme de phrases verbales descriptives conjuguées au présent de l'indicatif ${ }^{31}$. II en résulte une sorte de construction plus ou moins abstraite d'un idéal, non plus à atteindre mais accompli, comme nous pouvons le voir en Fig. 1. Ce choix du singulier défini, qui correspond certes à l'ambition de placer «l'élève au centre du système éducatif $»^{32}$, semble dans ce texte exclusif de l'enseignant et pourtant injonctif envers lui, voire exclusif des autres élèves. En revanche, axé sur les compétences du S4C, chaque item décrit y est indiqué comme enseignable et un but à atteindre pour tous, au maximum en fin de scolarité obligatoire : ici, la description vis-à-vis de l'objectif commun est interprétée comme un modèle institutionnel générique, et non un distributif de chaque individu, mais auquel chacun peut s'identifier plus facilement qu'avec des phrases au pluriel.

\subsubsection{LES POSTURES D'ÉLĖVES \\ DE BUCHETON ET SOULÉ}

II serait intéressant de comparer la manière dont les chercheurs en sciences de l'éducation mettent en mots leur(s) concept(s) d'élève(s) dans plusieurs articles de recherche ou d'ouvrages différents, mais pour les besoins de cette étude, nous réduisons la voilure en évoquant la manière dont l'élève est modélisé chez Bucheton et Soulé (2009), des travaux de recherche utilisés en formations d'enseignants, qui montrent, à l'instar de Meirieu, que l'hétérogénéité des élèves est un atout.

Puisque « le métier d'enseignant fait appel à des gestes professionnels ajustés », ces chercheurs proposent des outils d'analyse inspirés de leurs observations sur le terrain en mettant en évidence des postures d'enseignants corrélées aux postures d'élèves, et créent un modèle théorique correspondant à une catégorisation succincte $^{33}$, cinq postures génériques qui décrivent la manière dont « les élèves » (toujours désignés au pluriel avec une détermination définie) s'engagent ou non dans la tâche en fonction de leurs habitus, mais qui peuvent évoluer en fonction des gestes professionnels de l'ensei- gnant : des postures à la dénomination évocatrice dites " première », « ludique-créative », « réflexive », de refus » et « scolaire». II n'est nullement question de juger ni la personnalité, ni le caractère, ni les capacités cognitives de l'enfant, qui n'est mentionné que par l'analyse de son rapport au savoir, sa réaction à la posture de l'enseignant et aux situations de classe, comme nous pouvons le voir dans la synthèse proposée en Fig. 1. Chaque posture comporte ses versants positifs et négatifs, et les élèves qui réussissent le mieux disposent en toute logique d'une gamme plus variée et savent en changer selon les circonstances de l'apprentissage. Les chercheurs invitent les enseignants à conscientiser et à composer avec le déjà-là des élèves, qu'il s'incarne dans une forme de conformité scolaire ou de résistance à celle-ci.

\subsection{L'ÉLĖVE DANS LES REPRÉSENTATIONS DES ENSEIGNANTS EN FORMATION}

Nous avons travaillé autour de cette définition/caractérisation de l'élève avec plusieurs groupes d'enseignants en 2019-2020 : expérimentés, débutants ou étudiants de Master MEEF. L'échantillon d'environ 80 personnes peut paraître faible mais certains éléments sont apparus : comme nous pouvons le lire en Fig. 1, si les enseignants débutants, oubliant tout à coup leur bagage théorique, ont eu tendance à se focaliser sur des traits assez stéréotypés et très subjectifs en raison de leur manque d'expérience et leurs propres difficultés, que nous synthétiserons en bons élèves dociles vs élèves craints, en n'évoquant pas du tout les autres, les étudiants qui préparent le CRPE utilisent le lexique et les concepts attendus au concours, miment les discours institutionnels et la littérature de recherche. II est toutefois plus surprenant que les étudiants stagiaires semblent les mettre de côté quelques mois plus tard, sitôt sur le terrain.

De leur côté, les enseignants expérimentés ont plutôt deux représentations presque opposées, entre ceux qui mettent également en avant de manière subjective deux types d'élèves, de la même manière que les débutants, en y ajoutant le critère socioculturel de contexte familial défavorable, et ceux qui mettent en relation les différents comportements scolaires avec leurs contenus d'enseignement et l'ajustement de leur pédagogie. En cela, ils se rapprochent des postures d'élèves décrites dans la littérature de recherche évoquée plus haut, mais pensent aussi à prendre en compte les absentéistes. En revanche, l'ensemble des enseignants de terrain soulignent l'importance d'un cadre structuré sur lequel l'élève peut s'appuyer pour grandir, que la responsabilité soit scolaire ou familiale.

A l'image de l'expérience sémantique de Labov en

30 Excepté dans l'introduction qui désigne le public concerné et met en avant l'importance de la prise en compte des hétérogénéités.

31 Ce constat fera l'objet d'une étude à part entière mais ce n'est pas notre propos ici.

32 Expression consacrée par la « loi Jospin » de 1989 et défendue dans plusieurs articles de Meirieu (2017).

33 D’autres chercheurs mettent en évidence près de 70 postures d'élèves différentes. 
1973 sur la question de l'indétermination référentielle entre le «bol » et la « tasse», dans quelle mesure le bon élève du programme scolaire, celui qui ne sait pas déjà et a besoin de l'école pour progresser dans tous les domaines, indépendamment du déjà-là de ses compétences et de ses carences, devient-il le mauvais élève de l'enseignant?

\section{UNE IMAGE GESTALTIQUE DE L'ÉLĖVE IDÉAL}

Comme nous avons pu le voir en Fig. 1, les traits contextuels relevés ${ }^{34}$ construisent l'isotopie d'une image gestaltique d'élève modèle, à laquelle sont renvoyés les élèves réels, par le biais de l'espace d'ancrage médiatisé par le bulletin scolaire. Nous nous sommes donc demandé, sans pour autant confondre sens et référence (Kleiber 1997), si l'addition des traits sémantiques relevés pouvaient correspondre à des référents réels, et nous constatons plusieurs éléments, que nous avons schématisés en Fig.2.

Lorsque la désignation est objective, i.e. correspondant aux définitions des dictionnaires positionnées à la base de la pyramide, chaque NP d'élève lui correspond ce rôle, puisque tous ne parviennent pas à réussir.

Ensuite, les représentations des candidats au concours corroborent les modèles théoriques, incluent l'ensemble des élèves mais uniquement au sein des classes, donc oublient les absents, qui ne peuvent, de fait, s'approcher de l'idéal. Au milieu de notre synthèse, nous avons situé le modèle institutionnel présenté par le Socle, l'élève qui a atteint tous les objectifs, mais dans la réalité, tous les élèves du bas de cette échelle n'y parviennent pas, et dans ce texte, ni l'enseignant ni sa famille ne sont sollicités dans sa réussite. Nous retrouvons donc nos stéréotypes exclusifs, et l'effectif englobant les bons élèves diminue d'autant plus. Et enfin, l'élève des bulletins scolaires, en tous les cas lorsque le $N_{\mathrm{e}}$ est exprimé : l'élève évalué l'est en fonction de ce qui lui permet d'appartenir ou non à cette classe-type d'élève qui possède toutes les qualités attendues, mais nous avons vu précédemment que la majorité de ces qualités ne sont pas enseignées, dépendent de lui-même et surtout de son milieu socioculturel, et donc de ses parents.

En effet, dans les appréciations où apparaît le $N_{e}$ nous avons observé que son emploi contient une subjectivité masquée de l'énonciateur, à induire de sa situation d'énon-

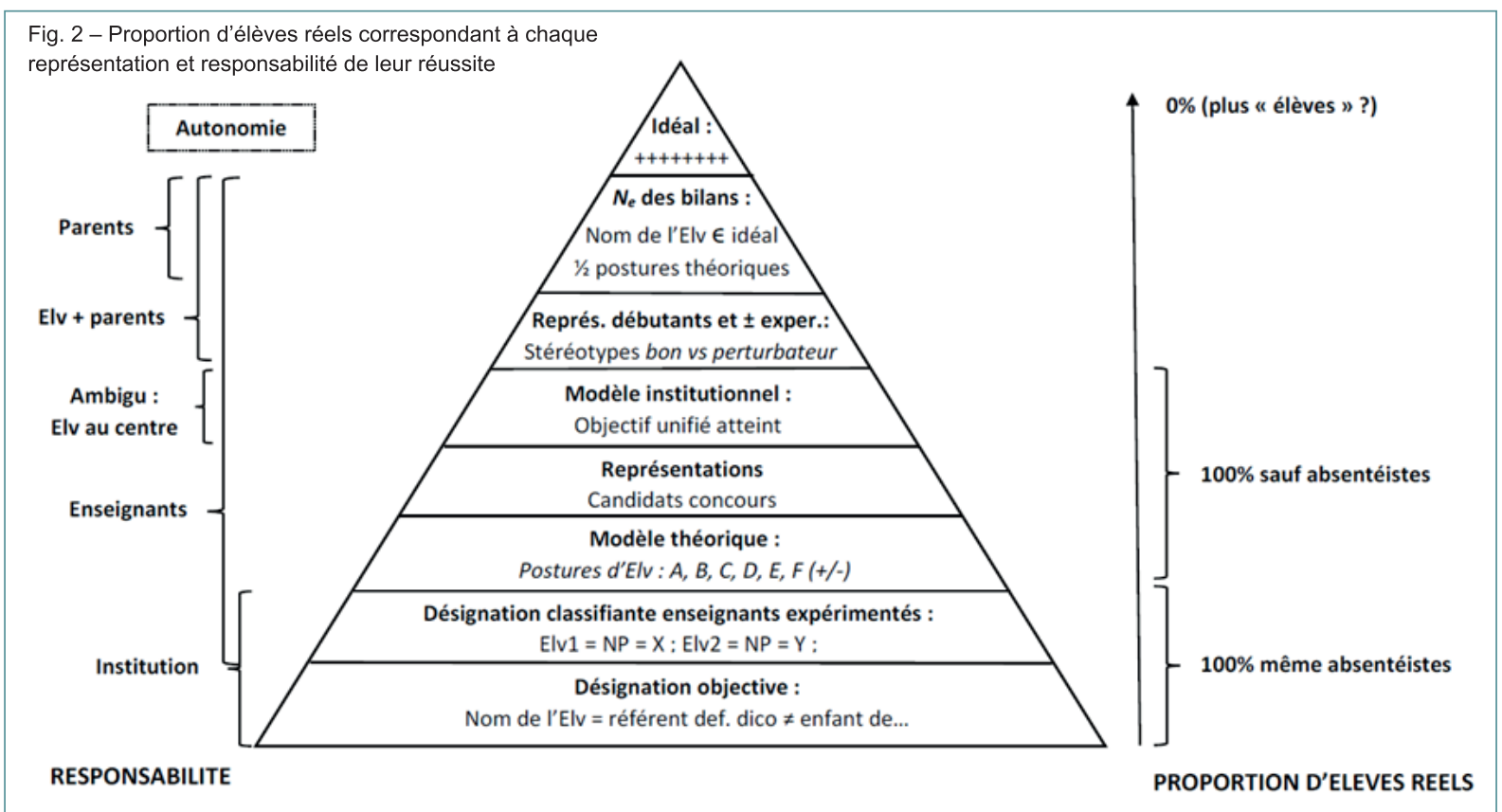

et ce dernier n'est pas considéré comme enfant d'Untel, soit 100\% des élèves. A l'inverse, plus on se rapproche du haut de la pyramide, avec au sommet l'élève idéal, plus le nombre d'élèves susceptibles de l'atteindre diminue, et la responsabilité de sa réussite - et corolairement de son échec scolaire - est diluée et attribuée à ses parents et/ou à l'élève lui-même. Deuxièmement, la désignation classifiante des enseignants expérimentés prend en considération l'ensemble des élèves, tout en les étiquetant selon leur profil, même les absentéistes, mais dans ce cas soulignent également les failles de l'Institution dans ciation et de la valeur subjective des termes choisis, même si les marques de première personne ne sont pas visibles dans son énoncé. D’autre part, dans ces appréciations sélectionnées, il s'adresse au parent, voire à ses pairs, pour comparer cet élève aux autres élèves de la classe en tant qu'il correspond à une catégorie-type d'élève.

Ensuite, ce qui est majoritairement indiqué comme positif est davantage lié à la personnalité de l'individu qu'à ses compétences scolaires en construction ; on le félicite pour ce qu'il est naturellement ou culturellement, pour les conséquences positives de la bonne éducation

34 Sèmes micro-génériques pour la lisibilité de l'ensemble. 
qui lui a pré-enseigné les codes, et non réellement pour les efforts fournis en classe, qu'il s'agit toujours de poursuivre ou intensifier sans nécessairement recevoir une indication du comment. A contrario, s'il ne mérite pas, s'il n'atteint pas ce « rôle d'élève », par la non-association attributive explicite avec ce terme ou sa négation, ce qui est négatif va dans le sens d'une injonction - sous forme de conseil ou d'ordre plus affirmé... aux parents - à être ou devenir à l'image du pendant positif de ce qui est décrit comme comportement scolaire négatif.

En réalité, lorsqu'on observe ce qu'implique l'image dessinée par l'utilisation de ce vocable, c'est que, paradoxalement, c'est l'élève qui a le plus besoin de l'école, celui qui doit atteindre le modèle institutionnel grâce à tous les traits inhérents à ce statut, qui se retrouve exclu de cette dénomination dans le contexte des bilans périodiques, comme s'il ne la méritait pas, par opposition à celles et ceux qui la méritent :

«Le sens d'une expression linguistique est constitué par des traits auxquels doit satisfaire une entité pour être désignée par cette expression linguistique (...) [qui] détermine par avance quels segments peuvent être désignés par elle et lesquels sont exclus ». (Kleiber 1997)

Pour risquer une analogie et pasticher Henri Frei et sa Grammaire des fautes (1929), imaginons, à l'instar des chercheurs en sciences de l'éducation, une grammaire de l'élève, qui en étudierait l'usage correct et les écarts par rapport à la norme véhiculée par certains enseignants dans leurs écrits. En effet, Frei étudie comment les fautes répondent à des exigences du langage, en quoi elles sont conditionnées par son fonctionnement. II part du principe que le locuteur qui commet un écart attend quelque chose de la langue qu'il n'y trouve pas. De la même manière, un élève qui fait des erreurs (scolaires ou comportementales) exprime un besoin, un ennui, une incomplétude à combler.

En face de lui, le prototype d'élève idéal, L'Emile évoqué en 2.3.1, qui n'existe que si l'on additionne toutes les qualités décrites par les actants de la communauté éducative, et dont le référent réel n'existe pas et ne peut exister. En effet, non seulement certains critères s'opposent - entre les injonctions officielles, les modèles théoriques, les contraintes concrètes et budgétaires de la réalité, entre les enseignants eux-mêmes, qui n'ont pas tous la même conception du métier - il correspondrait à un être, non plus en processus constant d'élévation, mais un être élevé, à l'aspect accompli, qui n'aurait plus besoin d'être élève de quiconque, mais, tel le sage revenu du Mondes des Idées dans l'allégorie de la Caverne, enseignant à son tour?

\section{CONCLUSION}

En conclusion, l'élève, tel qu'il est caractérisé dans les bilans périodiques envoyés aux familles, au-delà de ses traits inhérents par l'addition de ses atouts personnels validés par l'enseignant, l'élève qui « cocherait toutes les cases » dans une réalité rêvée telle qu'elle est esquissée par cette constellation qualitative, possèderait tous les traits sémantiques d'une créature idéale qui n’a pas ou plus besoin de ses professeurs pygmalions, mais surtout de bons créateurs qui récoltent finalement les éloges qui leur sont indirectement destinés. Par conséquent, si l'on poursuit l'idée que l'élève idéal des bulletins scolaires est affranchi de l'enseignant, l'utilisation du mot dans ce contexte professionnel, qui scelle pourtant un par un les paliers dont ce dernier est le promoteur, serait, sans le vouloir, en contradiction avec ses traits définitoires initiaux car, par implicature, exclusifs de l'enseignant - et même du reste de la classe - dont il devient, par comparaison, le maître-étalon.

\section{BIBLIOGRAPHIE}

Aristote, 1991[329 et 323 av. J.-C.]. Rhétorique, Le Livre de poche.

Austin, J. L., 1970. Quand dire, c'est faire, Paris, Seuil. Benveniste, E., 1966, 1974. Problèmes de linguistique générale 1 et 2, Paris : Gallimard.

Beyssade, C. (dir.), 2020. Les implicatures, au-delà du sens littéral, Iste Editions.

Bucheton, D., Soule, Y., 2009. «Les gestes professionnels et le jeu des postures de l'enseignant dans la classe: un multi-agenda de préoccupations enchâssées », Éducation et didactique URL : < http://journals.openedition.org/educationdidactique/543 >

Buvet, P.-A., 2009. « Détermination prédicative et déterminant zéro », Synergies Tunisie $n^{\circ} 1$, p. 145-158.

Descles, J-P., 2008. Opérations de prédication et de détermination, Lidil, URL : <http://journals.openedition.org/ lidil/2689> ; DOI : <https://doi.org/10.4000/lidil.2689>.

El Hadi, S., 2011. Dans le genre « peut mieux faire » : le bulletin scolaire, Linx. URL : <http://journals.openedition.org/linx/1413>.

Frei, H., 2011[1929]. La Grammaire des fautes, PUR.

Guiraud, P., 1960. Problèmes et méthodes de la statistique linguistique, Kluwer Academic Publishers.

Hawkins, J. A., 1978. Definiteness and Indefiniteness : A Study in Reference and Grammaticality Production, Croom Helm, Londres.

Heim, I., 1991. Artikel und Definitheit », in A. VON STECHOW et D. WUNDERLICH (dir.), Semantik : ein internationales Handbuch des zeitgenössischen Forschung, p. 487-535, de Gruyter, Berlon.

Kleiber, G., 1997. Sens, référence et existence : que faire de l'extra-linguistique?. In: Langages, $31^{\mathrm{e}}$ année, $n^{\circ} 127,1997$. Langue, praxis et production de sens. pp. 9-37; URL : <https://www.persee.fr/doc/ lgge_0458-726x_1997_num_31_127_2123>

Kleiber, G., 2009. D'un contexte à l'autre : aspects et dimensions du contexte. In: L'Information Grammaticale, N. 123, 2009. pp. 17-32. URL : <http://www.persee.fr/doc/ igram_0222-9838_2009_num_123_1_4061>.

Krazem, M., 1996. Le mot autonomie dans les projets éducatifs des municipalités : tentative de définition, Linx. URL : <http://journals.openedition.org/linx/1151>. 
L'elève des bulletins scolaires : le spectre sémantique d'un modèle utopique

Labov, W., 1973. The boundaries of words and their meanings, dans C.-J. Bailey et R. Shuy, New Ways of Analyzing Variation in English, Washington D.C., Georgetown University Press.

Larousse, consulté en ligne sur <https://www.larousse. $\mathrm{fr} />$, Société Editions Larousse.

Lebart, L., Pincemin B., Poudat, C., 2019. Analyse des données textuelles, PUQ.

Maingueneau, D., 2000[1998]. Analyser les textes de communication, Paris, Nathan.

Mayaffre, D., 2008. De l'occurrence à l'isotopie, in Syntaxe et sémantique $n^{\circ} 9$, Presses universitaires de Caen.

Meirieu, Ph., 2017. Ne pas renoncer à mettre l'élève au centre du système, Antidote $\mathrm{n}^{\circ} 25$ in Cahiers pédagogiques, HS n46. URL : <https://www.cahiers-pedagogiques.com/Ne-pas-renoncer-a-mettre-l-eleve-au-centre-du-systeme>.

MINISTERE DE L'EDUCATION NATIONALE, 2015. Socle Commun de connaissances, de compétences et de culture, décret n²015-372 du 31-3-2015 - J.O. du 2-4-2015.

Mortureux, M.-F., 1997. La lexicologie entre langue et discours Paris, SEDES, Coll. Campus.

Rastier, F., 2009[1997]. Sémantique interprétative, PUF.
Rastier, F., 2004. Enjeux épistémologiques de la linguistique de corpus, Texto ! URL : <http://www.revue-texto.net/index.php?id=543>.

Rey, A., 2000[1992]. Dictionnaire historique de la langue française, Paris : Le Robert

Riegel, M. Pellat, J.-C., Rioul, R., 2018[1994], Grammaire Méthodique du Français, 7e ed. PUF.

Roegiers, X., 2004. L'école et l'évaluation : des situations pour évaluer les compétences des élèves, De Boeck.

Roussarie, L., 2017. Sémantique formelle, Vol. 1, Introduction à la grammaire de Montague, Language Science Press.

Searle, J. R., 1972. Les Actes de langage, essai de philosophie du langage, Paris, Hermann.

Searle, J. R., 1982. Sens et expression, Ed. Minuit, Coll. Le sens commun.

TLFi : Trésor de la langue Française informatisé, URL : <http://www.atilf.fr/tlfi>, ATILF - CNRS \& Université de Lorraine.

Vygotski, L., 1985. Pensée et Langage Terrains, Éditions Sociales.

Return to front page $\uparrow$ 